\title{
Thermal Effusivity Determination of Carbon Fibre Reinforced Polymers by means of Active Thermography
}

\author{
by J. Suchan* and G. Hendorfer**
}

\author{
* University of Applied Sciences Upper Austria, Wels, Austria, josef.suchan@fh-wels.at \\ ** Josef Ressel Centre for Thermal NDE of Composites, School of Engineering, University of Applied Sciences \\ Upper Austria, Wels, Austria, guenther.hendorfer@fh-wels.at
}

\begin{abstract}
We present a new imaging approach to determine porosity in Carbon Fibre Reinforced Polymers by Active Thermography in the reflection mode. The IR-radiation is excited with help of a semiconductor laser. We use rectangular pulses for the excitation light - either single pulses or a sequence of a couple of pulses - and measure the succeeding temperature transients. These signals are transferred to the frequency domain by means of a discrete Laplace transformation. The evaluation of the thermal effusivity is done by a linear fitting process which gives unequivocal results with comparatively small error bars. The method is fast, robust and insensitive to inhomogeneous illumination. The results compare well with prior experiments carried out with ultrasonic-testing, X-Ray-Computed Tomography or other approaches of thermography, where the thermal diffusivity has been determined.
\end{abstract}

\section{Introduction}

Active Thermography is increasingly used as a method for non-destructive testing in recent years. Because it is a fast, non-contact and non-destructive method, it has entered the field of industrial applications [1]. Its main applications are in the field of the detection of defects, like voids, cracks, inclusions of foreign materials and many more. The method, however, is used also for the visualization and assessment of material properties - at least qualitatively. In carbon fibre reinforced plastics (CFRP) a material property of uttermost importance is the porosity, which appears as unavoidable sideeffect of the production process. Porosity can be considered as inclusion of gas-filled voids with typical diameters in the $\mu \mathrm{m}$-regime [2]. Since mechanical properties of CFRP such as transverse tensile strength as well as interlaminar shear strength decrease with increasing porosity $[3,4]$, the latter must be measured and evaluated after the production process. At present this is mainly done by means of ultrasonic testing [5] in the aviation industry.

It has been shown, however, that porosity in CPRP can be measured by means of Active Thermography as well [6]. Although the single pores cannot be detected because their size is clear below the spatial resolution limit of infraredcameras, the effect of porosity on the material properties like heat conduction coefficient $k$, density $\rho$ and specific heat capacity $c$ can be measured, as long as the material fulfils the criteria of homogeneous materials $[7,8]$. In a qualitative study of Grinzato et.al. [9] it was shown, that thermal diffusivity images, obtained by Active Thermography agree well with images, obtained by ultrasonic C-scan. Zalameda and Winfree [10] characterized simulated porosity in graphite composites using a phase lag technique to calculate the thermal diffusivity. A more advanced model which takes the microstructure into account was introduced as 'dethermalization theory' by Ringermacher [11]. The latter is derived from an effective medium theory developed by Mori-Tanaka [12] and takes the influence of the pore shape on the heat flux into account. We obtain information about the shapes of the pores by means of X-ray computed tomography (XCT)-investigations [13]. Representative data of pore size and pore shape are obtained by averaging over pore distributions obtained from XCTimages of our CFRP-samples. In order to correlate porosity to thermal diffusivity and effusivity, which are obtained from Thermography-experiments, the Maxwell-Garnett-Approximation [14] is used.

Our experiments have been performed by means of laser-excited pulsed thermography, since the completion of the experiments including the time for a data-evaluation can be done very fast then. A high testing speed, on the other hand, is a preferred approach of our industrial partners, where short testing times can be used as competitive advantage. The measurements can be done on the rear side of the sample, opposite of the excitation (transmission mode) or on the front side (reflection mode). Transmission mode measurements work very well with respect to reproducibility and accuracy, in particular if Parker's method [15] or the Linear Diffusivity Fitting (LDF)-method [16] are used. In industrial applications, however, reflection mode measurements are preferred in most cases. Unfortunately, the results in reflections mode measurements scatter much more as compared to transmission mode measurements, at least, if material properties are under investigation. As preferred methods for the former Thermal Signal Reconstruction (TSR) [17] in combination with fitting procedures [18] are used frequently. The limitations and shortcomings of those methods as compared to the LDFmethod in transmission mode measurements are discussed in [19] and suggest methodological improvements. 
We present a new method, well suited for reflection mode measurements, for the determination of the effusivity of CFRP or polymers in general. The method is based on laser excited pulsed thermography, using a semiconductor laser with a wavelength of $808 \mathrm{~nm}$. The light is modulated according to a square wave signal. The thermal transients are transferred to the frequency domain by means of a numerical Laplace transformation. After normalization of the Laplacetransformed signal the effusivity of the illuminated samples can be determined without curve fitting and independent of the value of heat transfer. The method, however, requires a linear fitting in the frequency domain, which gives unequivocal results. We call the method Linear Effusivity Fitting (LEF). It works for a sample width bigger than $2 \mathrm{~mm}$.

The validity of the method has been tested with help of simulations, which served as artificial measurement signals as well as experiments on CFRP-samples. The CFRP samples have also been measured by X-ray Computed Tomography, which served as a reference method for a porosity determination. The examinations indicate that our proposed new approach may serve as a fast, robust and accurate method for the determination of thermal properties which seems well suited for industrial applications. Since we determine effusivity, we have a better sensitivity for porosity contrary to measurements of thermal diffusivity. As is shown below, also the error bars are smaller than in the case of diffusivity measurements.

\section{Principle of the method}

The measurements are done in reflection mode and the samples are excited by laser light. The advantage of using a laser is obviously that the shape and the duration of the pulses can be adjusted exactly by control voltages and thus the reproducibility of pulse parameters is much better as compared to other light sources like flashes or lamps. The method works in principle for single pulses. It is, however, advantageous to apply a sequence of some identical pulses in order to optimize the signal to noise ratio. Long pulse sequences would require long measurement time. Thus, a compromise between signal to noise ratio and measurement time has to be found.

The power density $q(\mathrm{t})$, applied to the sample is known as a function of time and is transferred from time domain to frequency domain by means of a Laplace transformation, giving $q(\mathrm{~s})$. The temperature transients $T(t)$, following the excitation pulses are measured by an infrared camera and also transformed to the Laplace domain, giving $\overline{T(s)}$. The Laplace transformations have to be discretized according to equation (1)

$$
\bar{F}(s)=\mathcal{L}\{f\}(s)=\eta \sum_{n=0}^{\infty} e^{-s n \eta} \cdot f(n \eta) ; \eta=\frac{1}{f_{\text {sample }}}
$$

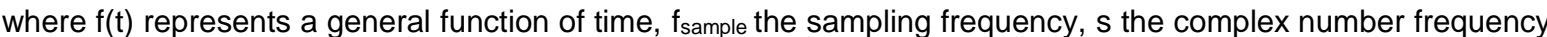
parameter and $F(s)$ the Laplace-transformed function of $f(t)$. In fig.1 (a) the power density of the excitation pulses as well as the temperature transients versus time are shown for single pulses obtained by simulations results. In fig. 1 (b) the corresponding Laplace-transformed functions are shown.

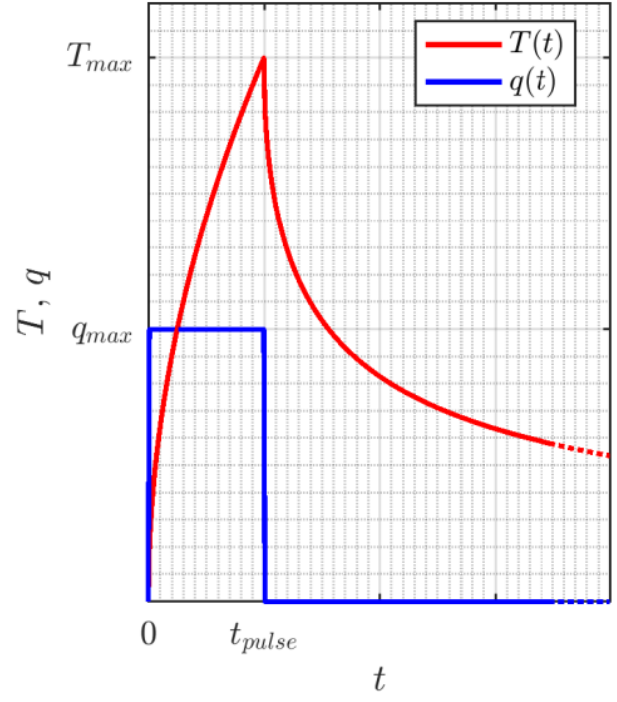

Fig. 1. (a) Power density of the exciting light $q$ as well as temperature response $T$ versus time in units of pulse time $t_{\text {pulse. }}$

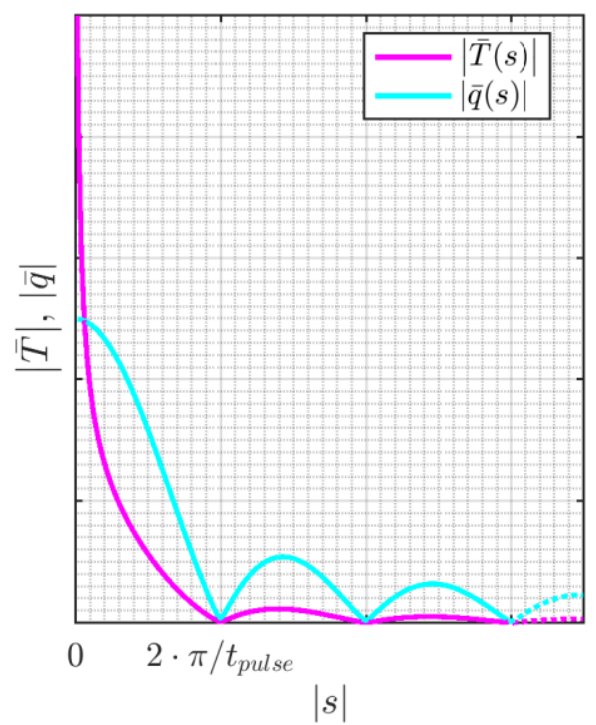

Fig. 1. (b) Laplace transformed signals $q^{-}$and $\bar{T}$ versus $s$ in units of $2 \pi / t_{\text {pulse }}$ 
By assuming that i) only signals on the sample surface are considered and that ii) thermal waves are used as basis function of the temperature transients [20] the ratio of $\overline{T(s)}$ and $\bar{q}(\mathrm{~s})$ can be expressed according to equation 2 .

$$
Z(x=0, s)=\frac{\bar{T}(0, s)}{\bar{q}(s)}=\frac{1}{e \cdot \sqrt{s}}
$$

where $e$ is the thermal effusivity, given as $e=(k \rho c)^{0.5}$. In fig. 2 (a) $Z(s)$ for several values for the effusivity is given versus $\mathrm{s}$, in fig. 2 (b) $\mathrm{Z}(\mathrm{s})$ is given versus $\mathrm{s}^{-0.5}$. In this plot a linear dependence appears, and the slope is equal to $1 / \mathrm{e}$.

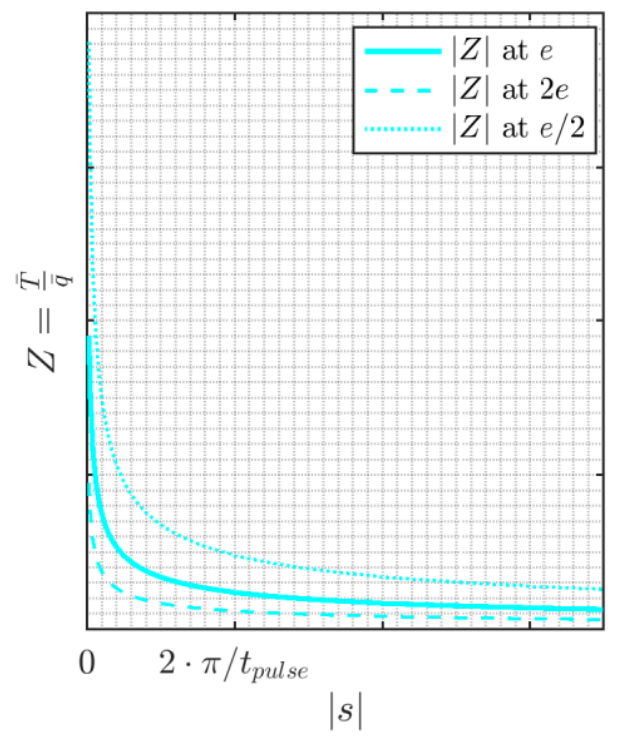

Fig. 2. (a) $Z(s)$ versus $s$ in units of $2 \pi / t_{\text {pulse. }}$

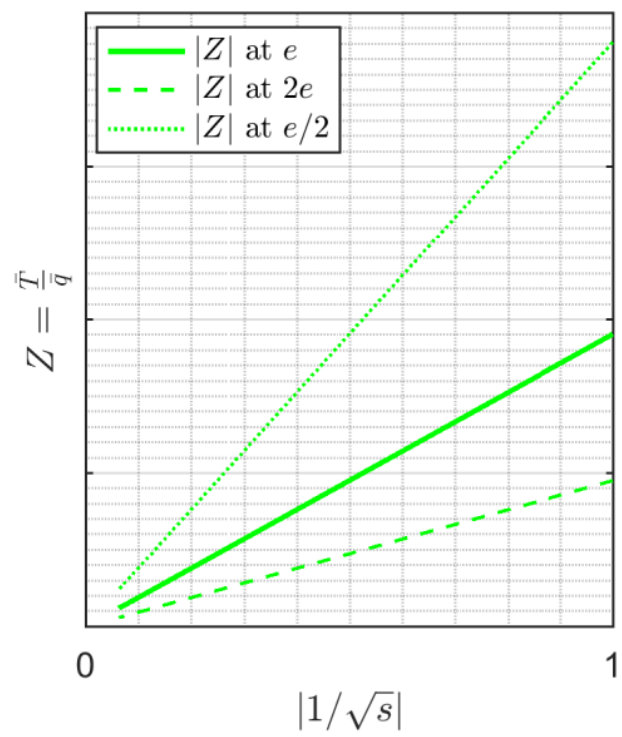

Fig. 2. (b) $Z$ (s) versus $s^{-0.5}$.

\section{Samples}

For this study, a prepreg fabric denoted as CYCOM 970/PWC T300 $3 \mathrm{k}$ was used. This type of prepreg is a CFRP. The samples were fabricated by using twenty ply plain weave style laminate. Each ply has a nominal thickness of 0,2159 $\mathrm{mm}$. The total thickness of all samples ranges from about $4.25 \mathrm{~mm}$ up to 4.83 . The area of each specimen has a size of about $40 \mathrm{~mm} \times 20 \mathrm{~mm}$. Fig 3. (a) shows an image of one of the specimens.

All specimen have previously been investigated by Active Thermography, more precisely the LDF- and the TSRmethod. The specimen have also been examined by means of X-ray computed tomography with an accuracy of an edge length of $10 \mu \mathrm{m}$ per volume element (voxel). Fig. 3. (b) shows a porosity map based on an XCT evaluation of a part of a specimen. Bright regions yield a high porosity, whereas darker regions denote a lower porosity. Fig. 3. (c) shows a threedimensional isometric view on a cubic section of a specimen. The grey structures denote the pores of a sample. The edge length of the cube is $4 \mathrm{~mm}$. The data used to create this image were also obtained from XCT-data.

A sound sample of CFRP without inclusions or porosities has a thermal conductivity of $\mathrm{k}=0.7 \mathrm{~W} \mathrm{~m}^{-1} \mathrm{~K}^{-1}$, a density of $\rho=1570 \mathrm{~kg} \mathrm{~m}^{-3}$ and a specific heat capacity of $c=1200 \mathrm{~K} \mathrm{~kg}^{-1} \mathrm{~K}^{-1}$ [19]. This yields a thermal effusivity of $1148 \mathrm{~W} \mathrm{~m}^{-2} \mathrm{~K} \mathrm{~s}^{-0.5}$ and a thermal diffusivity of $\alpha=3.74 \times 10^{-7} \mathrm{~m}^{2} \mathrm{~s}^{-1}$. 


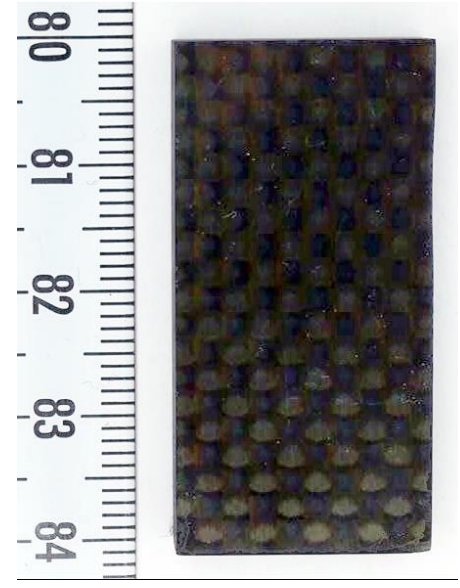

(a) Specimen

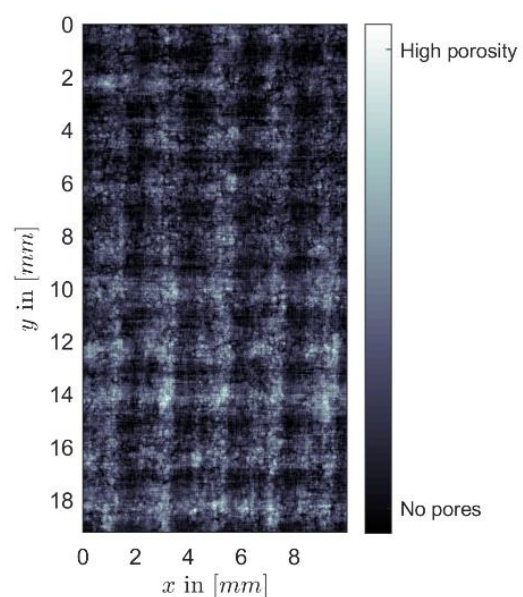

(b) Porosity map of a specimen

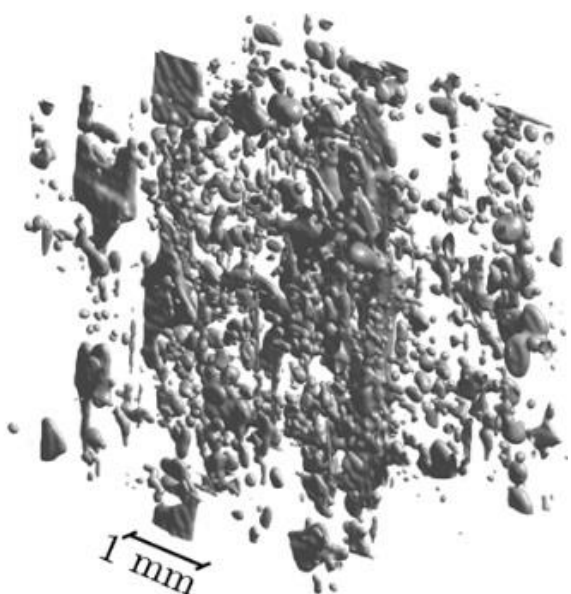

(c) 3D-view of porosity data

Fig. 3. Images showing the specimen used in the experiments. Image (a) shows one of the specimen next to a tape measure labelled in centimetres. Image (b) and (c) show a two- and three-dimensional view of the pores, respectively. The pore data were obtained by $X$-ray computed tomography.

\section{Experimental setup}

Fig. 4 shows a schematic diagram of the experimental setup. A computer-controlled semiconductor laser thermally excites a specimen for a time of $5 \mathrm{~s}$. To improve a homogeneous excitation of the specimen, the laser beam is guided through a diffusor. The laser excitation causes the specimen to leave its state of thermal equilibrium, resulting in a temperature increase at the surface of the specimen. After a duration of $5 s$, the laser excitation is stopped and the temperature on the surface of the specimen starts to decrease. The transient behaviour of the temperature at the surface of the specimen is recorded by an infrared camera. The recorded thermal images are finally evaluated by means of LEF.

The laser, that was used in the experiment is a Lumics LU0808D300 with a nominal operating power of $P=30 \mathrm{~W}$ and a wavelength of $\lambda=808 \mathrm{~nm}$. The camera is a FLIR X8400SC with a resolution of $1280 \times 1024$ pixels and a thermal sensitivity $<25 \mathrm{mK}$. The camera was set to a sample rate of $f=50 \mathrm{~Hz}$ and to an integration time of $2 \mathrm{~ms}$. The region of interest $(\mathrm{ROI})$ for the measurements covers the full sample. For each frame the average temperature of the ROI was calculated and stored for later post-processing.

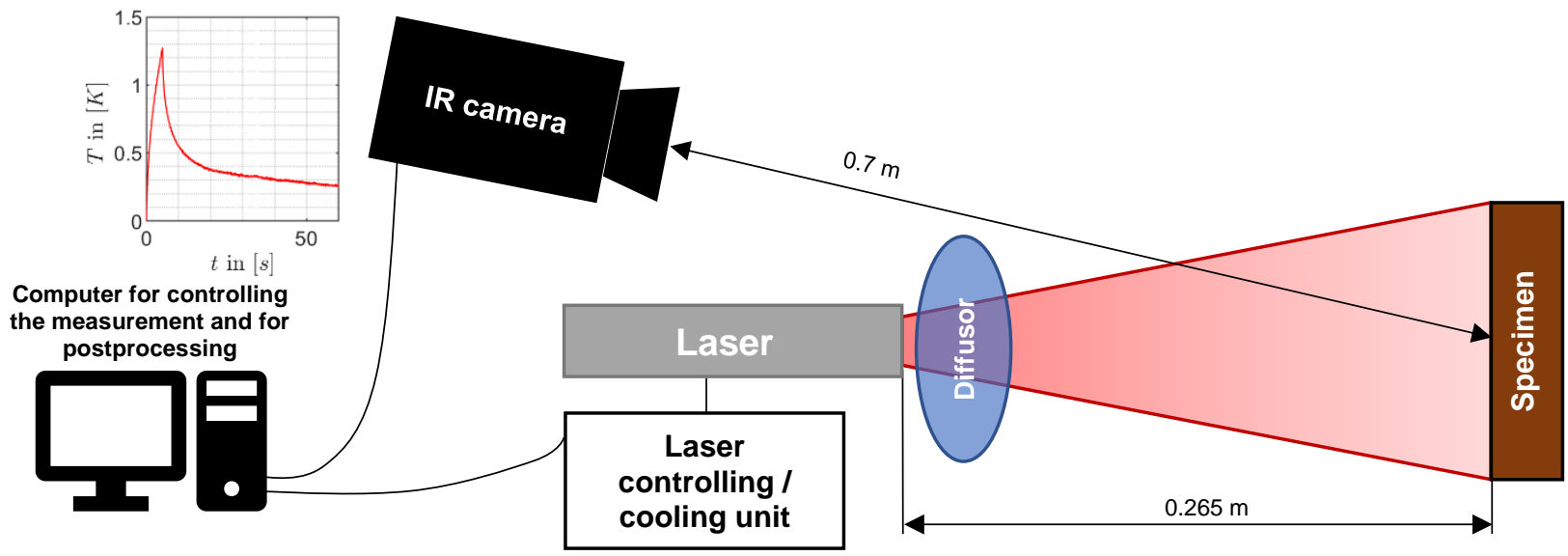

Fig. 4. Schematic diagram of the experimental setup. 


\section{Results}

\subsection{Simulation results}

Prior to the actual measurements, a simulation with finite-element (FEM) software was performed. The goal of this simulation was to test the LEF-algorithm with simulation data. The simulation consists of a one-dimensional virtual specimen with a fixed width and fixed material parameters for the thermal conductivity $k$, the density $\rho$ and the heat capacity c. These three parameters yield a thermal diffusivity e. In the simulation a heat pulse was applied to the virtual specimen and the resulting temperature at the surface of the specimen was recorded. In a next step, the LEF-algorithm was used, to obtain the specimen's thermal effusivity from the temperature transient, which was successful.

In the next stage it was determined whether a limitation for the thickness of the virtual specimen exists. In order to verify the result for this problem, another FEM simulation was created using the same input data as in the previous simulation, with one exception: The sample thickness was altered for each step and the thermal effusivity of the sample was determined using the LEF.

Fig. 5 shows the result of this simulation. It is visible, that although the basic equation for LEF is derived from the heat transfer equation for a semi-infinite body, the LEF can also be used for finite specimens down to a minimal sample thickness of $2 \mathrm{~mm}$. If the sample thickness is below $2 \mathrm{~mm}$, the error in the determination of the thermal effusivity increases significantly.

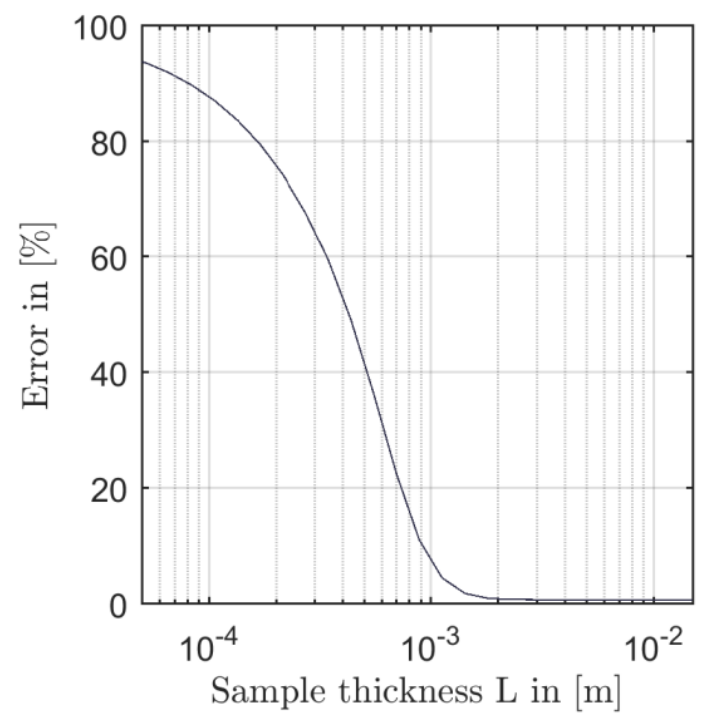

Fig. 5. Error in the thermal effusivity depending on the sample thickness $L$.

\subsection{Experimental results}

As described in section "Experimental setup", each sample has been evaluated by means of LEF, to determine its thermal effusivity. The thermal effusivity for every single sample has been determined for at least 3 times. Fig. 6 shows the results of the measurement of the thermal effusivity versus porosity $\phi$. The results have been normalized to the result of an effusivity measurement for a sample with a porosity of $\phi=0 \%$. The thermal effusivity of a sample with a porosity of $\phi=0 \%$ is $e=1148 \mathrm{~W} \mathrm{~m}^{-2} \mathrm{~K} \mathrm{~s}^{-0.5}$. The figure shows a decrease of the thermal effusivity as the porosity of the specimen increase.

Another value, that reflects the thermal properties of a sample in a more decent way than the porosity, is the diffusion time to. The diffusion time is the time required for a heat wave to propagate through a sample. The diffusion time depends on the specimen's material properties, especially the thermal diffusivity $\alpha$ and the sample thickness $L$. It is defined as

$$
t_{D}=\frac{L^{2}}{\alpha}
$$

The evaluated samples have also previously been tested by means of Linear Diffusivity Fit (LDF) [16, 19]. The results from the evaluation with LDF and the results of the measurement values obtained by LEF can be found in Table 1. The results for the thermal effusivity e of the samples versus the diffusion time $t_{D}$ are shown in Fig. 7. This figure also shows a good correlation between the diffusion time and the determined thermal diffusivity. 


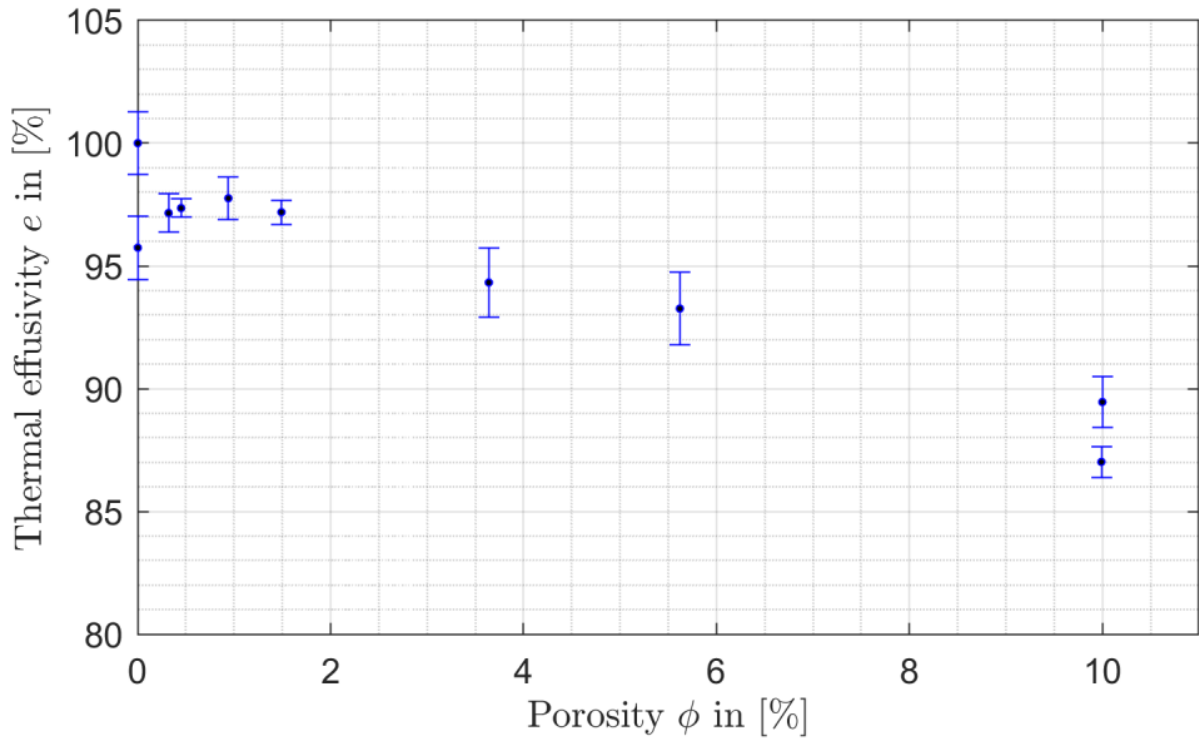

Fig. 6. Nominal thermal effusivity e versus sample porosity $\Phi$.

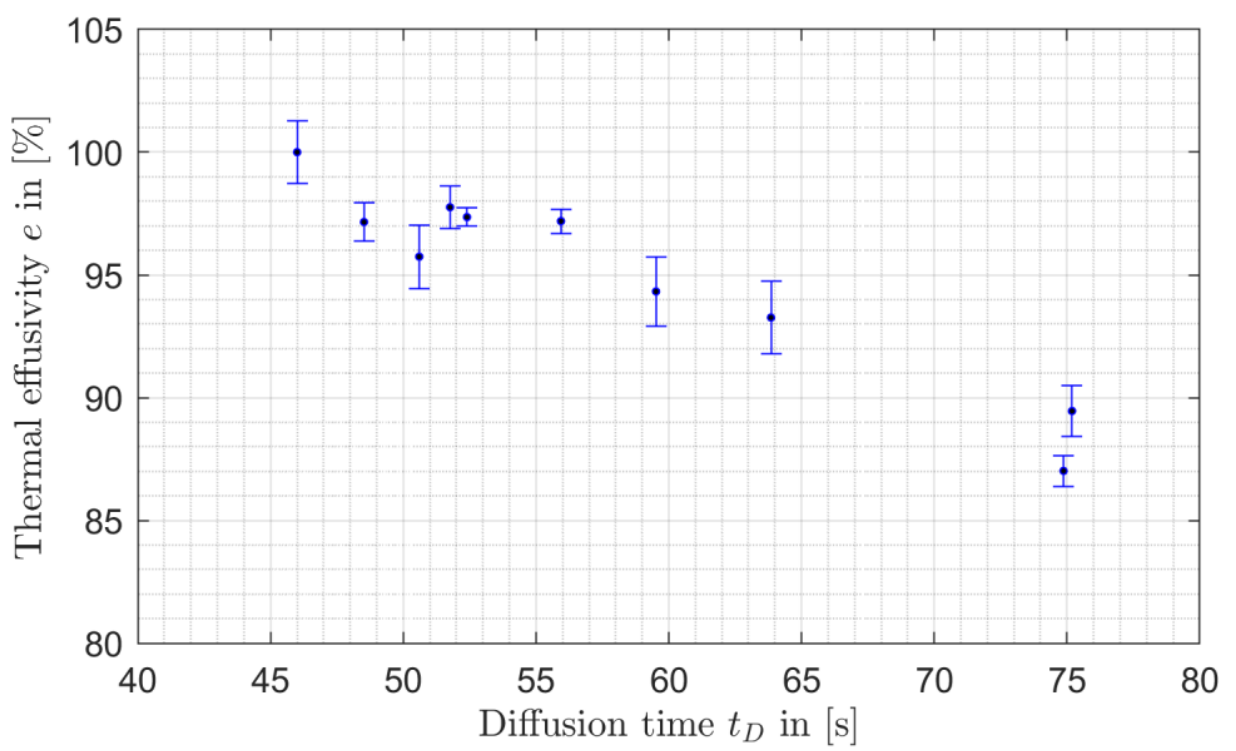

Fig. 7. Nominal thermal effusivity e versus diffusion time $t_{D}$.

Table 1. Values for porosity $\Phi$, sample width $L$, diffusion time to and results from thermal effusivity measurements obtained by LEF. Sample 1 was used as reference sample with porosity $\phi=0 \%$.

\begin{tabular}{|c|c|c|c|c|}
\hline Sample No. & Porosity & Sample width & Diffusion time & Thermal effusivity \\
\hline & $\boldsymbol{\Phi}$ in [\%] & L in [mm] & t in [s] $_{\text {[ }}$ e in [\%] of reference effusivity \\
\hline 1 & 0 & 4.25 & 46 & 100.0 \\
\hline 2 & 0 & 4.34 & 50.6 & 96.1 \\
\hline 3 & 0.32 & 4.32 & 48.52 & 95.0 \\
\hline 4 & 0.45 & 4.36 & 52.4 & 93.8 \\
\hline 5 & 0.94 & 4.36 & 51.76 & 95.9 \\
\hline 6 & 1.49 & 4.51 & 55.94 & 94.4 \\
\hline 7 & 3.64 & 4.51 & 59.52 & 93.1 \\
\hline 8 & 5.62 & 4.7 & 63.85 & 86.2 \\
\hline 9 & 9.99 & 4.82 & 74.87 & 85.5 \\
\hline 10 & 10.0 & 4.83 & 75.19 & \\
\hline
\end{tabular}




\subsection{Effusivity imaging of a sample}

The thermal effusivity of each sample in section 5.2 was determined by recording the mean temperature across an ROI on the sample and applying the LEF to the recorded mean temperature. It is also possible to decrease the size of this ROI down to a single pixel and then determine the thermal effusivity for every pixel on the sample. Fig. 8 (a) shows the temperature on sample 7 after a duration time of $t=3 \mathrm{~s}$. Fig. 8. (b) shows the inherent thermal effusivity image. The ROI is situated in the centre of the sample and has a size of about $16 \mathrm{~mm} \times 16 \mathrm{~mm}$. A pixel has an edge length of $200 \mu \mathrm{m}$.

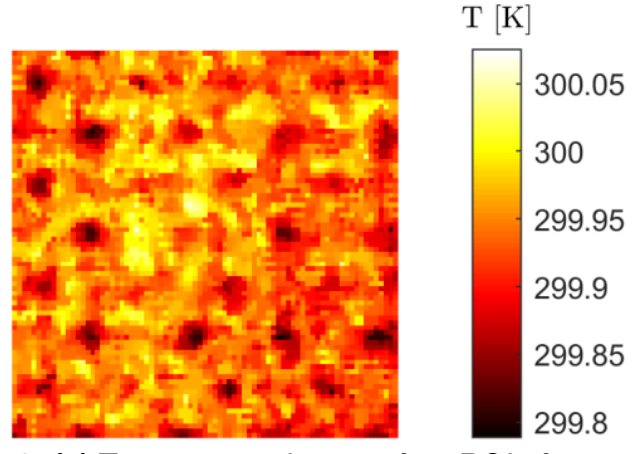

Fig. 8. (a) Temperature image of an ROI of $16 \times 16$ $\mathrm{mm}$ on sample after a duration of 3 seconds.

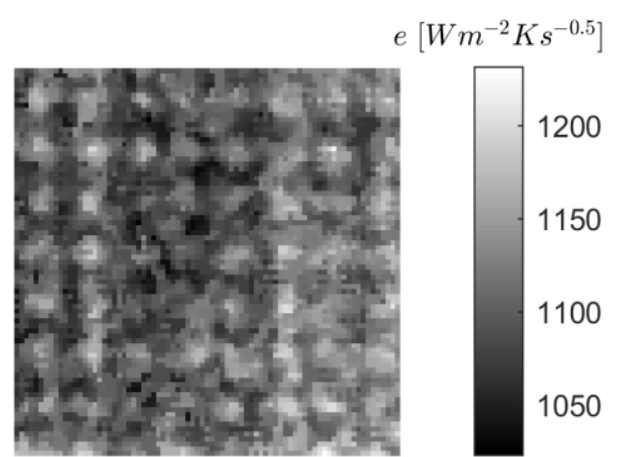

Fig. 8. (b) Image of the thermal effusivity of the same ROI as shown in Fig. 8. (a).

\section{Summary}

The LEF-approach of thermal effusivity determination is a fast and robust method which can be used to determine porosity in CFRP quantitatively. Porosity values, which have been determined in this way are in good agreement to former Thermography-measurements performed according to the TSR- and the LDF-approach. They are in good agreement to ultrasonic- and XCT-measurements as well. The evaluation is done in the frequency domain, because the signal-noise ratio is better as compared to the time domain. Effusivity images of the sample surface can be generated. They are insensitive to effects due to inhomogeneous illumination. The error bars of the porosity levels are comparable to those of ultrasonic testing. The drawback of the method is that emissivity of the specimen must be known or be the same for all specimen investigated.

\section{Acknowledgement}

The financial support by the Austrian Federal Ministry of Science, Research and Economy and the National Foundation for Research, Technology and Development is gratefully acknowledged.

\section{REFERENCES}

[1] Maldague X., "Theory and Practice of Infrared Technology for Nondestructive Testing". Wiley, New York, 2001

[2] Campbell F., "Structural Composite Materials". ASM International, Ohio, 2010

[3] Ghiorse S., "Effect of void content on the mechanical properties of carbon/epoxy laminates". SAMPE Quarterly, 24, pp. 54-59, 1993

[4] Olivier P., Cottu J., Ferret B., "Effects of Cure Cycle Pressure and Voids on some Mechanical Properties of Carbon/Epoxy Laminates". Composites Vol. 26, Issue 7, pp. 509-515, 1995.

[5] Daniel I., Wooh S., Komsky I., "Quantitative Porosity Characterization of Composite Materials by means of Ultrasonic Attenuation Measurements". Journal of Nondestructive Evaluation, Vol. 11(1), pp. 1-8, 1992.

[6] Conolly M., "The Measurement of Porosity in Composite Materials using Infrared Thermography". Journal of Reinforced Plastics and Composites, Vol. 11(12), pp. 1367-1375, 1992.

[7] Kerrisk J., "Thermal Diffusivity of Heterogeneous Materials". Journal of Applied Physics, Vol. 42(1), pp. 267-271, 1971.

[8] Torquato S., "Random Heterogeneous Materials - Microstructure and Macroscopic Properties". Springer, New York, 2002

[9] Grinzato E., Marinetti S., Bison P., "NDE of Porosity in CFRP by Multiple Thermographic techniques". Proc. SPIE4710, Thermosense XXIV, Orlando, 2002.

[10] Zalameda J., Winfree W., "Thermal Diffusivity Measurements on Composite Porosity Samples". Review of Progress in Quantitative Nondestructive Evaluation, Vol. 9, 1990. 
[11] Ringermacher H., Howard D., Gilmore R., "Discriminating Porosity in Composites using Thermal Depth Imaging". AIP Conference proceedings, Vol. 615(1), pp. 528-535, 2002.

[12] Mori T., Tanaka K., "Average Stress in Matrix and Average Elastic Energy of Materials with Misfitting Inclusions". Acta Metallurgica, Vol. 21(5), pp. 571-574, 1973.

[13] Kastner J., Plank B., Salaberger D., Sekelja J., "Defect and porosity Determination of Fibre Reinforced Polymers by X-ray Computed Tomography". $2^{\text {nd }}$ International Symposium on NDT in Aerospace, p. 12, 2010.

[14] Kaviany M., "Principles of Heat Transfer in Porous Media". Mechanical Engineering Series, $2^{\text {nd }}$ ed., Springer, New York, 1995.

[15] Parker, W., "Flash Method of Determining Thermal Diffusivity, Heat Capacity, and Thermal Conductivity". Journal of Applied Physics, Vol. 32(9), pp. 1679-1684, 1961.

[16] Hendorfer G., Mayr G., Zauner G., et al., "Quantitative Determination of Porosity by Active Thermography". Review of Progress in Quant. Nondestructive Evaluation, Vol. 26A, pp. 702-708, 2007.

[17] Shepard S., US Patent 6,516,084. 2000

[18] Balageas D., "Improvement of the Detection of Defects by Pulse Thermography Thanks to the TSR Approach in the Case of a Smart Composite Repair Patch". Proc. 10 ${ }^{\text {th }}$ Int. Edition Quantitative InfraRed Thermography, Vol.10, pp. 873-880, 2010.

[19] Mayr G., "Characterization of Porous Carbon Fiber Reinforced Polymer Components Using Optical Excited Pulse Thermography”. Doctoral dissertation, Linz, 2016.

[20] Almond D., Patel P., "Photothermal Science and Techniques". Chapman \& Hall, London, 1996 